\title{
sciendo
}

\section{The use of gamma irradiation to stimulate bioactive compound synthesis in Inonotus obliquus submerged cultures}

\author{
Alexandru Petre (D), \\ Mihaela Ene (D), \\ Daniel Constantin Negut $\mathbb{1}$, \\ Florentina Gatea $\mathbb{D}$, \\ Emanuel Vamanu
}

\begin{abstract}
Inonotus obliquus is a parasite on the birch and other trees and is also a well-known medicinal mushroom. Its sterile conk is highly sought for its bioactive compounds such as phenols, polysaccharides, triterpenoids, and steroids. It was traditionally used to treat various gastrointestinal diseases, viral and parasitic infections, to counteract the progression of cancers, and to stimulate the immune system. We used acute gamma irradiation, followed by short-term submerged cultivation, as an oxidative stress inducer to enhance the synthesis of mycelial metabolites. The $300 \mathrm{~Gy}$ and $400 \mathrm{~Gy}$ doses showed the best results across the whole experimental design. Each assayed criterion had a different corresponding optimal stimulation dose. In one experiment, sublethal doses of irradiation triggered the dry weight of the cultured mycelium to increase by $19.764 \%$. The free radical scavenging potential of the mycelium extracts increased by $79.83 \%$. The total phenolic content of mycelium extracts and culture broth increased by $55.7 \%$ and $62.987 \%$, respectively. The total flavonoid and sinapinic acid content of the broth increased by $934.678 \%$ and $590.395 \%$, respectively. As such, gamma irradiation pre-treatment of the mycelial inoculum proved an interesting, economically and environmentally effective tool for stimulating secondary metabolite synthesis in submerged mycelium cultures.
\end{abstract}

Keywords: Gamma irradiation $\bullet$ Chaga $\bullet$ Medicinal fungi $\bullet$ Metabolite synthesis $\bullet$ Bioactive compounds

\author{
A. Petre \\ Horia Hulubei National Institute for Physics and Nuclear \\ Engineering (IFIN-HH), Bucharest, Romania \\ and University of Agricultural Sciences and Veterinary \\ Medicine of Bucharest, Bucharest, Romania \\ E-mail: alexandru.petre@nipne.ro \\ M. Ene ${ }^{凶}$, D. C. Negut \\ Horia Hulubei National Institute for Physics and Nuclear \\ Engineering (IFIN-HH), Bucharest, Romania \\ E-mail: mene@nipne.ro \\ F. Gatea \\ National Institute of Research and Development \\ for Biological Science (INCDSB), Bucharest, Romania \\ E. Vamanu \\ University of Agricultural Sciences and Veterinary \\ Medicine of Bucharest, Bucharest, Romania
}

Received: 2 February 2021

Accepted: 7 June 2021

\section{Introduction}

The parasitic fungus Inonotus obliquus, also known as the Chaga mushroom (Hymenochaetaceae), grows on the trunk of birch and other trees in the circumboreal region of the northern hemisphere [1]), but does so very slowly, making it difficult to rely on the natural lifecycle of this mushroom for obtaining bioactive metabolites for industrial applications [2]. It produces various types of metabolites such as phenols, polysaccharides, triterpenoids, and steroids [3]. The phenolic compounds found in I. obliquus show great potential for the scavenging of free radicals [4]. Past research involving submerged cultures of this species has focused on the production of biomass [5] and the accumulation of phenolic compounds [6]. The great majority of research involving gamma radiation and mushrooms employs $\gamma$ rays as a conditioning agent with harvested mushrooms or extracts, aiming to increase nutrient or antioxidant levels through the breakdown of existing compounds $[7,8]$. In this study, we used acute gamma irradiation of submerged I. obliquus mycelium, followed by cultivation, as a tool to stimulate production of biologically active metabolites, in response to the oxidative stressor represented by radiation exposure, via a hormetic process. We monitored changes in

0029-5922 @ 2021 The Author(s). Published by the Institute of Nuclear Chemistry and Technology.

This is an open access article under the CC BY-NC-ND 4.0 licence (http://creativecommons.org/licences/by-nc-nd/4.0/). 
mycelial biomass and antioxidant levels, as well as the amount and class of compounds with antioxidant properties.

\section{Methods}

\section{Mycelium cultivation and irradiation}

I. obliquus (Ach. ex Pers.) Pilát (CBS 314.39) mycelium was purchased from Westerdijk Fungal Biodiversity Institute (Utrecht, The Netherlands) and grown on potato dextrose agar for seven days at $23.5^{\circ} \mathrm{C}$. Subsequently, $1 \mathrm{~cm} \times 1 \mathrm{~cm}$ agar sectors were each transferred to potato dextrose broth (PDB) seed culture medium. The mycelium was cultured at the same temperature, in $500 \mathrm{~mL}$ bottles with polytetrafluoroethylene (PTFE) membrane screw caps, agitated at $100 \mathrm{rpm}$ for 28 days, until it occupied the entire volume of the culture vessels. In order to ensure identical inoculum for all replicates, five such fully grown colonies were brought together in a sterile $3000 \mathrm{~mL}$ Erlenmeyer flask and triturated; and $400 \mathrm{~mL}$ of the homogenized mycelium was distributed back into the culture vessels.

The application of the oxidative stress induced by gamma radiation on the mycelium of I. obliquus, in a liquid medium, was performed separately on each culture vessel. Sublethal doses of 0,100, 200, 300, and 400 Gy were given to the triturated mycelium, according to the experimental design.

After the cultures were irradiated, each was used to seed five replicates of new PDB liquid cultures by transferring $80 \mathrm{~mL}$ of homogenized mycelium (a 20\% $\mathrm{v} / \mathrm{v}$ inoculum) to a total volume of $400 \mathrm{~mL}$ of new culture. Thus, the irradiation experiment had five doses with five replicates each, which were cultured for 16 days after being irradiated, under the same temperature and agitation parameters as before.

\section{Gamma radiation technology and dosimetry}

Irradiations were carried out using the Co-60 research irradiator GC-5000 (B.R.I.T., India), within the IRASM Radiation Processing Center, part of "Horia Hulubei" National Institute of Physics and Nuclear Engineering, Romania.

We used an alanine-EPR dosimetry system to evaluate doses. The irradiations were performed at an average dose rate of $0.8 \mathrm{~Gy} / \mathrm{s}$ with an associated relative uncertainty of $3 \%$. The dose uniformity ratio, defined as the ratio of the maximum to the minimum dose in a set of samples, was about 1.6. All doses are expressed as absorbed dose in water.

\section{Mycelial biomass and fermented broth harvesting}

16-day old cultures were harvested by separating the mycelial biomass from the fermented broth through a $500 \mathrm{~nm}$ stainless steel sieve, followed by further filtration of the broth through a $180 \mathrm{~mm}$ Nylon Net (Merck Millipore), under vacuum. The mycelia were then washed three times with deionized water and frozen at $-50^{\circ} \mathrm{C}$ before being freeze dried. Mycelial biomass was estimated by dry weight.

\section{Methanolic extraction of the dry mycelium}

$100 \mathrm{mg}$ of each irradiated mycelium was milled to a powder, distributed to $2 \mathrm{~mL}$ microtubes, and extracted two times in cold methanol. The dried biomass was kept in $2 \mathrm{~mL}$ of $80 \%$ cold methanol for $24 \mathrm{~h}$ at $-50^{\circ} \mathrm{C}$, and then the tubes were agitated on a horizontal shaker at $1300 \mathrm{rpm}$ for $30 \mathrm{~min}$ and centrifuged for $10 \mathrm{~min}$ at $14000 \mathrm{rpm}$. The two supernatants were saved and reunited in the end.

To remove lipids, each extract was washed three times with an equal volume of hexane, discarding the organic phase between washes. After removing the last volume of hexane, the extracts were evaporated at $40^{\circ} \mathrm{C}$ and then redissolved in $1 \mathrm{~mL}$ of $80 \%$ methanol on a horizontal shaker at $1300 \mathrm{rpm}$. Ultimately, the extracts were then centrifuged for $10 \mathrm{~min}$ at $14000 \mathrm{rpm}$ to remove any undissolved particles.

\section{Colorimetric assays}

Negative controls were prepared using distilled water or $80 \%$ methanol, depending on the sample solvent. Samples were reacted three times with the colorimetric reagents and quantified twice. Results were calculated as an average of these technical replicates.

\section{DPPH free radical scavenging assay}

The free radical scavenging potential of the mycelium extracts was determined using a modified DPPH (2,2-diphenyl-1-picryl-hydrazyl-hydrate) colorimetric method [9]. A $0.125 \mathrm{mM}$ DPPH (TCI Chemicals) solution was prepared using a 4:1 (v/v) methanol-PBS mixture, of which $295 \mathrm{~mL}$ were reacted with $5 \mathrm{~mL}$ of each extract. The reaction mixture was kept at room temperature, in darkness, for $30 \mathrm{~min}$. The absorbance of the reaction mixture was recorded at $517 \mathrm{~nm}$ using a SpectraMax i3x (Molecular Devices) plate reader.

The calibration curve $(0-500 \mathrm{mg} / \mathrm{mL})$ was plotted using ascorbic acid dissolved in $80 \%$ methanol as a standard. The free radical scavenging potential was expressed as milligram of ascorbic acid equivalent per milliliter.

\section{Total phenolic quantification assay}

Both extra- and intra-cellular total phenolic compounds (EPC and IPC) were quantified from culture broth and mycelium extract, respectively.

The total phenolic content was determined by colorimetric methods [10] with Folin-Ciocalteu reagent (Sigma-Aldrich). A 10-fold dilution of this reagent and a $7.5 \%(\mathrm{w} / \mathrm{v}) \mathrm{Na}_{2} \mathrm{CO}_{3}$ solution were prepared using deionized water. A $200 \mathrm{~mL}$ sample was mixed with $1 \mathrm{~mL}$ of the diluted Folin-Ciocalteu reagent, followed by $1 \mathrm{~mL}$ of sodium carbonate solution. The 
reaction mixture was kept at room temperature, in darkness, for $30 \mathrm{~min}$. The absorbance of the reaction mixture was recorded at $765 \mathrm{~nm}$ using a SpectraMax i3x plate reader.

The calibration curve $(0-200 \mathrm{mg} / \mathrm{mL})$ was plotted using gallic acid as a standard. The total phenols were expressed as microgram of gallic acid equivalent per milliliter.

\section{Total flavonoid quantification assay}

The total flavonoid content of the whole crude fermentation (culture) broth was determined using a modified aluminium chloride colorimetric method [11]. A 2.5\% (w/v) $\mathrm{AlCl}_{3}$ solution was prepared using $50 \%(\mathrm{v} / \mathrm{v})$ ethanol, of which $1 \mathrm{~mL}$ was mixed with an equal volume of a fermented broth sample. The reaction mixture was kept at room temperature, in darkness, for $30 \mathrm{~min}$. The absorbance of the reaction mixture was recorded at $420 \mathrm{~nm}$ using a SpectraMax i3x plate reader.

The calibration curve $(0-50 \mathrm{mg} / \mathrm{mL})$ was plotted using quercetin as a standard. The total flavonoids were expressed as microgram of quercetin equivalent per milliliter.

\section{Capillary zonal electrophoresis analysis of polyphenols}

\section{Sample preparation}

The fermentation broths were subjected to a solid phase extraction (SPE) procedure to obtain concentrated extracts in polyphenols. Chromabond cartridges (Macherey-Nagel, $500 \mathrm{mg}$ ) were used. The stationary phase of the cartridges was activated by successive washes with methanol and water, after which $30 \mathrm{~mL}$ of each sample was added. The elution of the polyphenols from the cartridges was performed with $10 \mathrm{~mL}$ of methanol. The extracted samples were evaporated to dryness in a turbovap (Eppendorf) at $30^{\circ} \mathrm{C}$, and the residue thus obtained was dissolved in $100 \mathrm{~mL}$ methanol, centrifuged, and injected into the capillary electrophoresis apparatus. The results are given as the average of three injections.

\section{Capillary zonal electrophoresis}

Polyphenols separation was performed on an Agilent CE system with a diode array detector. A standard bare fused-silica capillary (I.D. $50 \mathrm{~mm}$, effective length of $72 \mathrm{~cm}$ ) was used for separation. Before each sample migration, the capillary was washed with $0.1 \mathrm{M} \mathrm{NaOH}$, ultrapure water, and migration buffer. The sample was injected in hydrodynamic mode (3500 Pa, $12 \mathrm{~s}$ ) and the capillary was maintained at $30^{\circ} \mathrm{C}$. The migration buffer consisted of a $50 \mathrm{M}$ sodium tetraborate and $0.9 \mathrm{mM}$ sodium dodecyl sulfate (SDS) solution, with the $\mathrm{pH}$ adjusted to 9.15 using $1 \mathrm{M} \mathrm{HCl}$. The applied voltage was $30 \mathrm{kV}$ and the detection was performed in the $200-360 \mathrm{~nm}$ range [12]. The quantification of the compounds was performed by measurements at a $280 \mathrm{~nm}$ wavelength. The data have been processed using the ChemStation (Agilent) application for data analysis.

\section{Results and discussion}

\section{Analysis of the effect of gamma irradiation on mycelial biomass}

The gamma irradiation of mycelial cultures and subsequent biomass analysis were performed in duplicate, each experiment containing 25 shake flask cultures (five doses with five replicates each). Results from both experiments are shown separately.

For the first experimental run, after irradiation and 16 days of shake flask culture, the dry mycelial biomass presented an increase of 4.572-19.764\% in the irradiated experimental variants, depending on the dose, compared to the non-irradiated controls.

The statistical significance between the irradiated groups in terms of dry mycelium weight was evaluated by analysis of variance (Table 1 ). The result demonstrates that the differences are statistically highly significant $(F=12.520$, and $P<0.001)$.

To identify the groups between which these differences are found, and to ascertain the degree of significance for each comparison, the Tukey-Kramer post-hoc test was performed. The result showed very significant $(P<0.010)$ differences between the

Table 1. The one-way ANOVA of dry mycelium weight after irradiation and culture of the first experimental run

\begin{tabular}{ccccccc}
\hline Groups & Count & Sum & Average & Variance & Std. deviation & Std. error \\
\hline $0 \mathrm{~Gy}$ & 5 & 6.78 & 1.356 & 0.00468 & 0.068411 & 0.030594 \\
$100 \mathrm{~Gy}$ & 5 & 7.09 & 1.418 & 0.00342 & 0.058481 & 0.026153 \\
$200 \mathrm{~Gy}$ & 5 & 7.73 & 1.546 & 0.00753 & 0.086776 & 0.038807 \\
$300 \mathrm{~Gy}$ & 5 & 8.07 & 1.614 & 0.00863 & 0.092898 & 0.041545 \\
$400 \mathrm{~Gy}$ & 5 & 8.12 & 1.624 & 0.00433 & 0.065803 & 0.029428 \\
\hline
\end{tabular}

ANOVA single factor $(\alpha=0.01)$

\begin{tabular}{lccccc}
\hline Source of variation & SS & df & MS & $F$ & $P$-value \\
\hline Between groups & 0.286376 & 4 & 0.071594 & 12.52081147 & 0.000029182 \\
Within groups & 0.114360 & 20 & 0.005718 & & 430690161 \\
Total & 0.400736 & 24 & & & \\
\hline
\end{tabular}

SS, sum of squares; df, degrees of freedom; MS, mean square. 


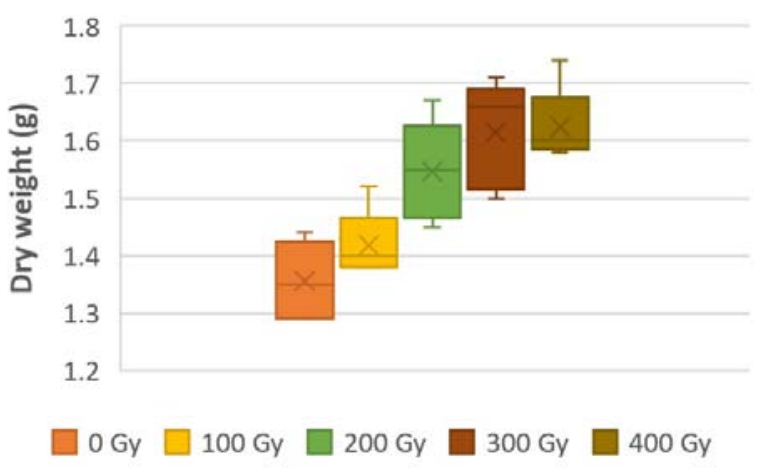

Fig. 1. The radiation dose-dependent distribution of dry mycelium weight per $400 \mathrm{~mL}$ of culture, in the first experimental run.

following groups: 0 Gy vs. 200, 300, and $400 \mathrm{~Gy}$; 100 Gy vs. 300 Gy and $400 \mathrm{~Gy}$.

The distribution of dry mycelium weight within and between irradiated groups is represented in Fig. 1 .

For the second experimental run, after irradiation and 16 days of shake flask culture, the dry mycelial biomass presented an increase of $8.586-16.630 \%$ in the irradiated experimental variants, depending on the dose, compared to the non-irradiated controls.

The statistical significance between the irradiated groups in terms of dry mycelium weight was evaluated by analysis of variance (Table 2). The result demonstrates that the differences are statistically highly significant $(F=13.286$ and $P<0.001)$.

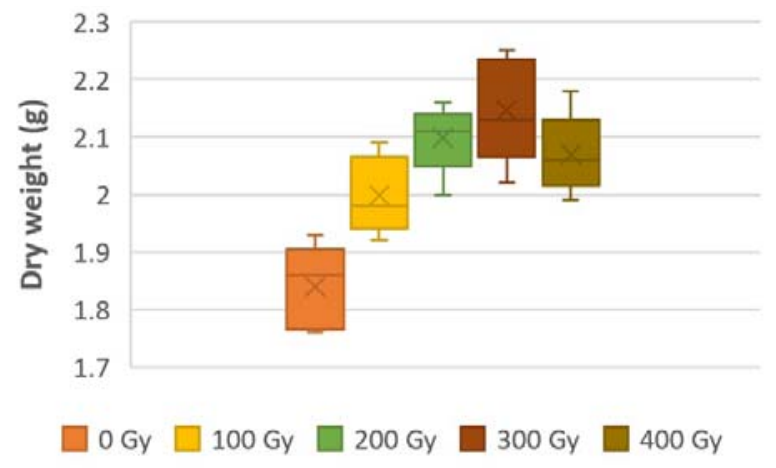

Fig. 2. The radiation dose-dependent distribution of dry mycelium weight per $400 \mathrm{~mL}$ of culture, in the second experimental run.

To identify the groups between which these differences are found, and to ascertain the degree of significance for each comparison, the TukeyKramer post-hoc test was performed. The result showed very significant $(P<0.010)$ differences between the following groups: 0 Gy vs. 200,300 , and 400 Gy. Significant $(P<0.050)$ differences were also found between the $0 \mathrm{~Gy}$ vs. $100 \mathrm{~Gy}$ groups; and 100 Gy vs. 300 Gy groups.

The distribution of dry mycelium weight within and between irradiated groups is represented in Fig. 2.

Table 2. The one-way ANOVA of dry mycelium weight after irradiation and culture of the second experimental run

\begin{tabular}{|c|c|c|c|c|c|c|}
\hline Groups & Count & Sum & Average & Variance & Std. deviation & Std. error \\
\hline $0 \mathrm{~Gy}$ & 5 & 9.20 & 1.840 & 0.00535 & 0.073144 & 0.032710 \\
\hline $100 \mathrm{~Gy}$ & 5 & 9.99 & 1.998 & 0.00452 & 0.067231 & 0.030066 \\
\hline $200 \mathrm{~Gy}$ & 5 & 10.49 & 2.098 & 0.00352 & 0.059330 & 0.026532 \\
\hline $300 \mathrm{~Gy}$ & 5 & 10.73 & 2.146 & 0.00843 & 0.091815 & 0.041060 \\
\hline$\underline{400 \mathrm{~Gy}}$ & 5 & 10.35 & 2.070 & 0.00490 & 0.07 & 0.031304 \\
\hline \multicolumn{7}{|c|}{ ANOVA single factor $(\alpha=0.01)$} \\
\hline Source of variation & SS & df & MS & $F$ & $P$-value & $F$ crit. \\
\hline Between groups & 0.284016 & 4 & 0.071004 & 13.28667665 & 0.000019303 & 2.866081402 \\
\hline Within groups & 0.106880 & 20 & 0.005344 & 0.10688 & & \\
\hline Total & 0.390896 & 24 & & & & \\
\hline
\end{tabular}

SS, sum of squares; df, degrees of freedom; MS, mean square.

Table 3. The one-way ANOVA of free radical scavenging potential of the mycelium extracts

\begin{tabular}{|c|c|c|c|c|c|c|}
\hline Groups & Count & Sum & Average & Variance & Std. deviation & Std. error \\
\hline $0 \mathrm{~Gy}$ & 5 & 1.3562 & 0.2712 & 0.0019 & 0.044382 & 0.019848 \\
\hline $100 \mathrm{~Gy}$ & 4 & 1.6489 & 0.4122 & 0.0011 & 0.034140 & 0.017070 \\
\hline $200 \mathrm{~Gy}$ & 5 & 2.1722 & 0.4344 & 0.0008 & 0.029030 & 0.012983 \\
\hline $300 \mathrm{~Gy}$ & 5 & 2.4386 & 0.4877 & 0.0025 & 0.050210 & 0.022455 \\
\hline$\underline{400 \mathrm{~Gy}}$ & 5 & 2.3953 & 0.4790 & 0.0014 & 0.038588 & 0.017257 \\
\hline \multicolumn{7}{|c|}{ ANOVA single factor $(\alpha=0.01)$} \\
\hline Source of variation & SS & $\mathrm{df}$ & MS & $F$ & $P$-value & $F$ crit. \\
\hline Between groups & 0.152096309 & 4 & 0.038024077 & 23.466281 & 0.0000003965 & 4.500257699 \\
\hline Within groups & 0.030787046 & 19 & 0.001620371 & & & \\
\hline Total & 0.182883355 & 23 & & & & \\
\hline
\end{tabular}

SS, sum of squares; df, degrees of freedom; MS, mean square. 


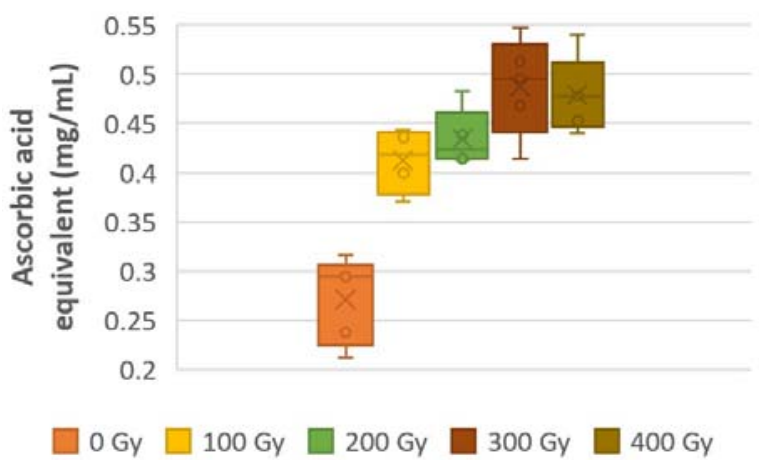

Fig. 3. The radiation dose-dependent distribution of free radical scavenging potential of the mycelium extracts.

Determination of the free radical scavenging activity

The DPPH free radical scavenging activity of the irradiated mycelium extracts showed a 51.99-79.83\% increase, depending on the dose, in comparison with non-irradiated control.

The statistical significance between the irradiated groups in terms of free radical scavenging potential of the mycelium extracts was evaluated by analysis of variance (Table 3). The result demonstrates that the differences are statistically highly significant $(F=23.466$ and $P<0.001)$.

To identify the groups between which these differences are found, and to ascertain the degree of significance for each comparison, the Tukey-Kramer post-hoc test was performed. The result showed very significant $(P<0.010)$ differences between the following groups: 0 Gy vs. 100, 200, 300, and $400 \mathrm{~Gy}$.

The distribution of free radical scavenging potential of the mycelium extracts within and between irradiated groups is represented in Fig. 3.

\section{Determination of the total phenolic content}

The total phenolic content of the mycelium methanolic extract increased by 28.4-55.7\%, depending on the dose, in comparison with non-irradiated controls.

The statistical significance between the irradiated groups in terms of total phenolic content of the mycelium extracts was evaluated by analysis of variance (Table 4). The result demonstrates that the

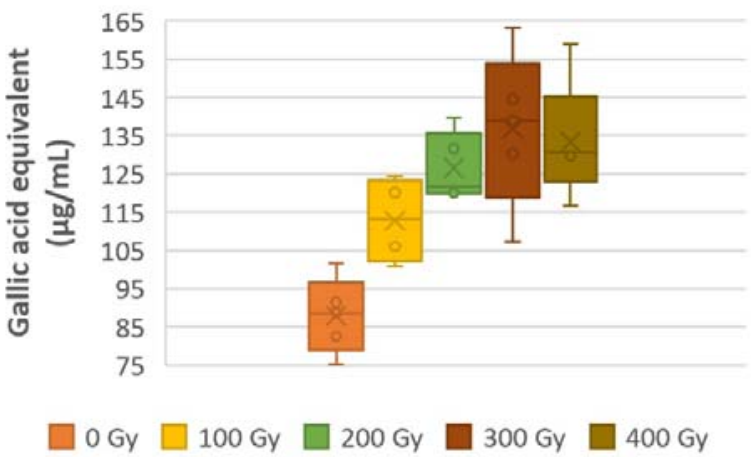

Fig. 4. The radiation dose-dependent distribution of total phenolic content of the mycelium extracts.

differences are statistically highly significant $(F=$ 10.167 and $P<0.001)$.

To identify the groups between which these differences are found, and to ascertain the degree of significance for each comparison, the Tukey-Kramer post-hoc test was performed. The result showed very significant $(P<0.010)$ differences between the following groups: 0 Gy vs. 200, 300, and $400 \mathrm{~Gy}$.

The total phenolic content of the mycelium extracts within and between irradiated groups is represented in Fig. 4.

Furthermore, the extra-cellular total phenolic content was also estimated by the same method for the crude fermentation broth. As with the mycelium extracts, the statistical significance between the irradiated groups was evaluated by analysis of variance (Table 5). The result demonstrates that the differences are statistically highly significant $(F=$ 15.102 and $P<0.001)$. Results showed an increase by $40.04-62.987 \%$, depending on the dose, in comparison with the non-irradiated control.

To identify the groups between which these differences are found, and to ascertain the degree of significance for each comparison, the Tukey-Kramer post-hoc test was performed. The result showed very significant $(P<0.010)$ differences between the following groups: 0 Gy vs. $300 \mathrm{~Gy}$ and $400 \mathrm{~Gy} ; 100 \mathrm{~Gy}$ vs. 200,300 , and 400 Gy. Significant $(P<0.050)$ differences were found between the 0 Gy vs. 200 Gy groups.

The total phenolic content of the fermented broths within and between irradiated groups is represented in Fig. 5.

Table 4. The one-way ANOVA of total phenolic content of the mycelium extracts

\begin{tabular}{cccrrrc}
\hline Groups & Count & Sum & Average & Variance & Std. deviation & Std. error \\
\hline $0 \mathrm{~Gy}$ & 5 & 439.4212 & 87.8842 & 98.0936 & 9.9042 & 4.4293 \\
$100 \mathrm{~Gy}$ & 4 & 451.3970 & 112.8492 & 124.0126 & 11.1361 & 5.5680 \\
$200 \mathrm{~Gy}$ & 5 & 632.8615 & 126.5723 & 76.2878 & 8.7343 & 3.9061 \\
$300 \mathrm{~Gy}$ & 5 & 684.2167 & 136.8433 & 417.0659 & 20.4222 & 9.1331 \\
$400 \mathrm{~Gy}$ & 5 & 667.4257 & 133.4851 & 238.70581 & 15.4501 & 6.9095 \\
\hline
\end{tabular}

ANOVA single factor $(\alpha=0.01)$

\begin{tabular}{lcrcccc}
\hline Source of variation & SS & df & MS & $F$ & $P$-value & $F$ crit \\
\hline Between groups & 7904.44478 & 4 & 1976.111195 & 10.16779312 & 0.00014191 & 4.500257699 \\
Within groups & 3692.651123 & 19 & 194.3500591 & & & \\
Total & 11597.0959 & 23 & & & & \\
\hline
\end{tabular}

SS, sum of squares; df, degrees of freedom; MS, mean square. 


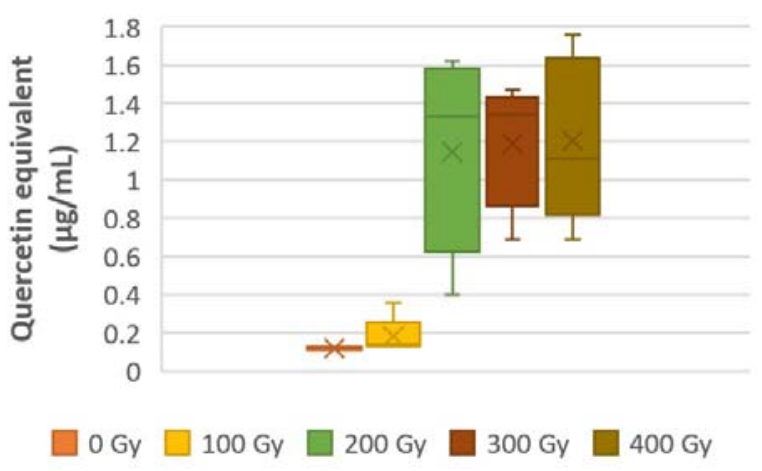

Fig. 5. The radiation dose-dependent distribution of total phenolic content of the fermented broths.

\section{Determination of the total flavonoid content}

The total flavonoid content of the crude culture broth increased by $26.6-934.678 \%$, depending on the dose, in comparison with non-irradiated controls.

The statistical significance between the irradiated groups in terms of total flavonoid content of the fermented broths was evaluated by analysis of variance (Table 6). The result demonstrates that the differences are statistically highly significant $(F=14.089$ and $P<0.001)$.

To identify the groups between which these differences are found, and to ascertain the degree of significance for each comparison, the Tukey-Kramer

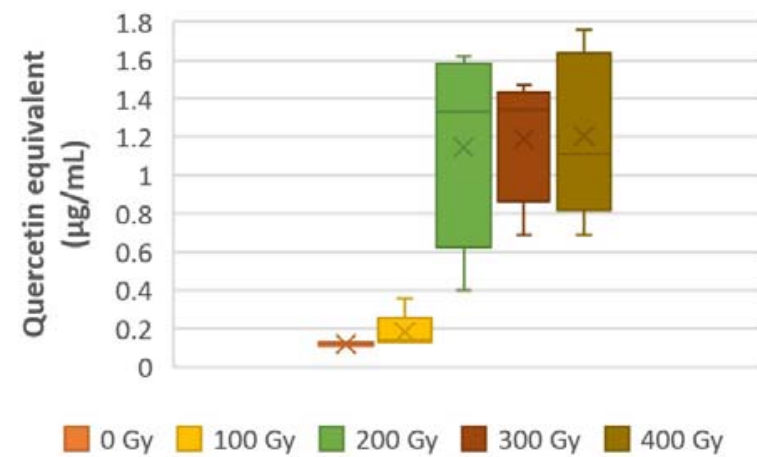

Fig. 6. The radiation dose-dependent distribution of total flavonoid content of the fermented broths.

post-hoc test was performed. The result showed very significant $(P<0.010)$ differences between the following groups: 0 Gy vs. 200,300 , and $400 \mathrm{~Gy}$; $100 \mathrm{~Gy}$ vs. 200, 300, and $400 \mathrm{~Gy}$.

The total flavonoid content of the fermented broths within and between irradiated groups is represented in Fig. 6.

\section{Capillary electrophoresis analysis of excreted compounds}

The concentrated fermentation broths were assessed by capillary electrophoresis using 14 standards, such as were rutin, naringenin, isoquercitrin, umbellifer-

Table 5. The one-way ANOVA of total phenolic content of the fermented broths

\begin{tabular}{|c|c|c|c|c|c|c|}
\hline Groups & Count & Sum & Average & Variance & Std. deviation & Std. error \\
\hline $0 \mathrm{~Gy}$ & 5 & 73.6308 & 14.72610 & 4.2755 & 2.0678 & 0.9247 \\
\hline $100 \mathrm{~Gy}$ & 5 & 72.1087 & 14.42170 & 0.4407 & 0.6639 & 0.2969 \\
\hline $200 \mathrm{~Gy}$ & 5 & 103.1155 & 20.62311 & 7.9304 & 2.8161 & 1.2594 \\
\hline $300 \mathrm{~Gy}$ & 5 & 111.8099 & 22.36199 & 3.9634 & 1.9908 & 0.8903 \\
\hline$\underline{400 \mathrm{~Gy}}$ & 5 & 120.0085 & 24.00170 & 15.6380 & 3.9545 & 1.7685 \\
\hline \multicolumn{7}{|c|}{ ANOVA single factor $(\alpha=0.01)$} \\
\hline Source of variation & SS & df & MS & $F$ & $P$-value & $F$ crit. \\
\hline Between groups & 389.6157862 & 4 & 97.403946550 & 15.10220151 & 0.000007715 & 4.430690161 \\
\hline Within groups & 128.9930431 & 20 & 6.449652157 & & & \\
\hline Total & 518.6088293 & 24 & & & & \\
\hline
\end{tabular}

SS, sum of squares; df, degrees of freedom; MS, mean square.

Table 6. The one-way ANOVA of total flavonoid content of the fermented broths

\begin{tabular}{lcccccc}
\hline \multicolumn{1}{c}{ Groups } & Count & Sum & Average & Variance & Std. deviation & Std. error \\
\hline $0 \mathrm{~Gy}$ & 5 & 0.5825 & 0.1165 & 0.00007 & 0.008539 & 0.003819 \\
$100 \mathrm{~Gy}$ & 5 & 0.9134 & 0.1826 & 0.00974 & 0.098740 & 0.044158 \\
$200 \mathrm{~Gy}$ & 5 & 5.7376 & 1.1475 & 0.26596 & 0.515721 & 0.230637 \\
$300 \mathrm{~Gy}$ & 5 & 5.9381 & 1.1876 & 0.10480 & 0.323743 & 0.144782 \\
$400 \mathrm{~Gy}$ & 5 & 6.0272 & 1.2054 & 0.18652 & 0.431886 & 0.193145 \\
\hline \multicolumn{2}{c}{ ANOVA single factor $(\alpha=0.01)$} & & \multirow{2}{*}{$F$ crit. } \\
\hline Source of variation & $\mathrm{SS}$ & $\mathrm{df}$ & $\mathrm{MS}$ & & & \\
\hline Between groups & 6.392584426 & 4 & 1.598146107 & 14.08987153 & 0.000012734 & 4.430690161 \\
Within groups & 2.268503447 & 20 & 0.113425172 & & & \\
Total & 8.661087873 & 24 & & & & \\
\hline
\end{tabular}

SS, sum of squares; df, degrees of freedom; MS, mean square. 
Table 7. The one-way ANOVA of the sinapinic acid content of the fermentation broths

\begin{tabular}{lcccrrr}
\hline \multicolumn{1}{c}{ Groups } & Count & Sum & Average & Variance & Std. deviation & Std. error \\
\hline $0 \mathrm{~Gy}$ & 5 & 40.0005 & 8.0001 & 25.7390 & 5.073365 & 2.536682 \\
$100 \mathrm{~Gy}$ & 3 & 51.3022 & 17.1007 & 26.4171 & 5.139765 & 3.634363 \\
$200 \mathrm{~Gy}$ & 2 & 58.7856 & 29.3928 & 304.6469 & 20.619987 & 14.580533 \\
$300 \mathrm{~Gy}$ & 3 & 91.1330 & 30.3776 & 18.1002 & 4.254438 & 3.008342 \\
$400 \mathrm{~Gy}$ & 1 & 55.2323 & 55.2323 & 0 & 0 & 0 \\
\hline
\end{tabular}

ANOVA single factor $(\alpha=0.01)$

\begin{tabular}{lcrrrrr}
\hline Source of variation & SS & df & MS & $F$ & $P$-value & $F$ crit. \\
\hline Between groups & 2466.721606 & 4 & 616.6804015 & 11.17539311 & 0.001532718 & 6.422085458 \\
Within groups & 496.6378863 & 9 & 55.18198737 & & & \\
Total & 2963.359492 & 13 & & & & \\
\hline
\end{tabular}

SS, sum of squares; df, degrees of freedom; MS, mean square.

one, cinnamic acid, chlorogenic acid, sinapinic acid, ferulic acid, kaempferol, luteolin, coumaric acid, quercetol, caffeic acid, and gallic acid.

Electrophoresis of the samples showed the presence of several compounds in the culture broths. However, not all of the samples could be analysed due to a clogging issue with the capillaries when migrating the extracts.

Naringenin was found at a concentration ranging from $4.94 \mathrm{mg} / 100 \mathrm{~mL}$ to $15.16 \mathrm{mg} / 100 \mathrm{~mL}$ of broth, across all the irradiated groups. After applying an analysis of variance, no statistical significance was found $(F=1.404$ and $P>0.100)$ linking the given radiation dose and increases in broth concentration of naringenin.

Isoquercitrin was found at a concentration ranging from $18.76 \mathrm{mg} / 100 \mathrm{~mL}$ to $131.27 \mathrm{mg} / 100 \mathrm{~mL}$ of broth, across all the irradiated groups. After applying an analysis of variance, no statistical significance was found $(F=2.044$ and $P>0.100)$ linking the given radiation dose and increases in broth concentration of isoquercitrin.

Chlorogenic acid was found at a concentration ranging from $3.96 \mathrm{mg} / 100 \mathrm{~mL}$ to $7.21 \mathrm{mg} / 100 \mathrm{~mL}$ of broth, across all the irradiated groups. After applying an analysis of variance, no statistical significance was found $(F=0.004$ and $P>0.100)$ linking the given radiation dose and increases in broth concentration of chlorogenic acid.

Luteolin was found at a concentration ranging from $2.15 \mathrm{mg} / 100 \mathrm{~mL}$ to $19.75 \mathrm{mg} / 100 \mathrm{~mL}$ of broth, across all the irradiated groups. After applying an analysis of variance, no statistical significance was found $(F=2.409$ and $P>0.100)$ linking the given radiation dose and increases in broth concentration of luteolin.

Sinapinic acid was found at a concentration ranging from $1.41 \mathrm{mg} / 100 \mathrm{~mL}$ to $55.23 \mathrm{mg} / 100 \mathrm{~mL}$ of broth, across all the irradiated groups. After applying an analysis of variance, we found differences that are statistically highly significant (Table 7).

The sinapinic acid content of culture broth showed a 113.756-590.395\% increase, depending on the dose, compared to the non-irradiated control.

To identify the groups between which these differences are found, and to ascertain the degree of significance for each comparison, the Tukey-Kramer



Fig. 7. The radiation dose-dependent distribution of sinapinic acid content of the fermentation broths.

post-hoc test was performed. The result showed very significant $(P<0.010)$ differences between the $0 \mathrm{~Gy}$ vs. 400 Gy groups. Significant $(P<0.050)$ differences were also found between the $0 \mathrm{~Gy}$ vs. $200 \mathrm{~Gy}$ and $300 \mathrm{~Gy} ; 100 \mathrm{~Gy}$ vs. $400 \mathrm{~Gy}$ groups.

The total sinapinic acid content of the fermentation broths within and between irradiated groups is represented in Fig. 7.

\section{Conclusion}

In this study, oxidative stress induced by gamma irradiation on the mycelial inoculum of the medicinal fungus I. obliquus, followed by shake flask cultivation, has shown a very significant increase in the free radical scavenging activity and levels of total polyphenols, in the methanolic mycelium extracts. The optimal dose, according to these assayed criteria, is $300 \mathrm{~Gy}$.

The fermentation broth of the cultures has shown a very significant increase in levels of total polyphenols and total flavonoids, corresponding to larger radiation doses. The optimal dose, in these instances, is $400 \mathrm{~Gy}$. The broths were further assayed using capillary electrophoresis. Naringenin, isoquercitrin, chlorogenic acid, luteolin, and sinapinic acid were found to be present, but only the latter showed statistically significant increases correlated to higher radiation doses; in this case, the optimal dose was $300 \mathrm{~Gy}$.

The total dry weight of the submerged cultured mycelia also showed a very significant increase after irradiation, with an optimal dose at $300 \mathrm{~Gy}$ 
in one experiment, and $400 \mathrm{~Gy}$ in another. Both experiments show a trend of higher mycelial mass correlated with increasing irradiation.

To summarize, the 300-400 Gy dose interval proved optimal for inoculum pre-treatment in a biotechnological process of I. obliquus submerged cultivation, in order to obtain a synergic effect on biomass and antioxidant properties. A 16 days cultivation period, supporting a fivefold multiplication of the inoculum's volume, is an efficient reaction window to develop and reveal the stimulation effect.

Other authors used oxidative stress to trigger the enhanced synthesis of diverse secondary metabolites synthesis. Zheng et al. [6] increased the production of phenolic compounds in I. obliquus by imposing oxidative stress caused by addition of $\mathrm{H}_{2} \mathrm{O}_{2}$ and/or arbutin. Similarly, Zheng et al. [2] evidenced the involvement of nitric oxide (NO) in modulating metabolism of polyphenols by the fungus I. obliquus under submerged culture conditions. The photosynthetic efficiency markedly decreased in gamma-irradiated Zygnema spp. alga, from $1 \mathrm{kGy}$ to $5 \mathrm{kGy}$, whereas antioxidant capacity significantly increased [13].

To our knowledge, this is the first work showing the use of sublethal doses of gamma irradiation as a pre-treatment for increasing biomass yield and for upregulating synthesis of biologically active metabolites in medicinal fungi. Since the treatment is applied to a small mycelium mass that is subsequently multiplied, such treatment is of particular interest for both fundamental biological processes and industrial potential. Medically potent compounds synthesis is yet to be investigated through this novel production system. The technique is both economically and environmentally effective in exploitation of endangered species and satisfying nutraceutical and pharmaceutical markets' demands for higher amounts of bioactive ingredients.

Acknowledgments. This work was supported by a grant of the Romanian Ministry of Research and Innovation, CCCDI - UEFISCDI, project no. 171PED/2017 (PN-III-P2-2.1-PED-2016-0132) and 5PCCDI/2018 (PN-III-P1-1.2-PCCDI-2017-0323), within PNCDI III.

\section{ORCID}

M. Ene (D) http://orcid.org/0000-0002-2715-8866

F. Gatea (D) http://orcid.org/0000-0002-6617-9811

D. C. Negut (D) http://orcid.org/0000-0002-7910-8801

A. Petre (D http://orcid.org/0000-0002-3512-2871

E. Vamanu (D) http://orcid.org/0000-0002-3376-2058

\section{References}

1. Lee, M. W., Hur, H., Chang, K. C., Lee, T. S., Ka, K. H., \& Jankovsky, L. (2008). Introduction to distribu- tion and ecology of sterile conks of Inonotus obliquus. Mycobiology, 36(4), 199-202.

2. Zheng, W., Miao, K., Zhao, Y., \& Zhang, M. (2009a). Nitric oxide mediates fungalelicitor-enhanced biosynthesis of antioxidant polyphenols in Inonotus obliquus in submerged cultures. Microbiology, 155, 3340-334. DOI: 10.1099/mic.0.030650-0.

3. Kim, Y. J., Park, J., Min, B. S., \& Shim, S. H. (2011). Chemical constituents from the sclerotia of Inonotus obliquus. J. Korean Soc. Appl. Biol. Chem., 54, 287-294. https://doi.org/10.3839/jksabc.2011.045.

4. Cui, Y., Kim, D. S., \& Park, K. C. (2005). Antioxidant effect of Inonotus obliquus. J. Ethnopharmacol., 96(1/2), 79-85. DOI: 10.1016/j.jep.2004.08.037.

5. Wang, Z. H., Huo, Y. F., Wang, B., \& Shen, J. W. (2006). Study on submerged cultures of Inonotus obliquus. Mycosystema, 25, 461-467. DOI: 10.4489/ MYCO.2008.36.4.199.

6. Zheng, W., Zhang, M., Zhao, Y., \& Wang, Y. (2009b). Accumulation of antioxidant phenolic constituents in submerged cultures of Inonotus obliquus. Biores. Technol., 100, 1327-1335. DOI: 10.1016/j. biortech.2008.05.002.

7. Kim, J. H., Sung, N. Y., Kwon, S. K., Srinivasan, P., Song, B. S., Choi, J. I., Yoon, Y., Kim, J. K., Byun, M. W., Kim, M. R., \& Lee, J. W. (2009). $\gamma$-Irradiation improves the color and antioxidant properties of Chaga mushroom (Inonotus obliquus) extract. J. Med. Food, 12(6), 1343-1347. DOI: 10.1089/jmf.2008.1281.

8. Fernandes, A., Antonio, A. L., Oliveira, M. B., Martins, A., \& Ferreira, I. C. (2012). Effect of gamma and electron beam irradiation on the physico-chemical and nutritional properties of mushrooms: a review. Food Chem., 135(2), 641-650. DOI: 10.1016/j.foodchem.2012.04.136.

9. Shen, Q., Zhang, B., Xu, R., Wang, Y., Ding, X., \& Li, P. (2010). Antioxidant activity in vitro of seleniumcontained protein from the Se-enriched. Bifodobacterium animalis 01. Anaerobe, 16, 380-386. DOI: 10.1016/j.anaerobe.2010.06.006.

10. Singleton, V., \& Rossi, J. (1965). Colorimetry of total phenolic compounds with phosphomolybdicphosphotungstic acid reagents. Am. J. Enol. Viticult., 16, 144-158.

11. Chang, C. C., Yang, M. H., Wen, H. M., \& Chern, J. C. (2002). Estimation of total flavonoid content in propolis by two complementary colorimetric methods. J. Food Drug Anal., 10(3), 178-182. DOI: 10.38212/2224-6614.2748.

12. Matei, A. O., Gatea, F., Teodor, E. D., \& Radu, G. L. (2016). Polyphenols analysis from different medicinal plants extracts using capillary zone electrophoresis (CZE). Rev. Chim., 67(6), 1051-1055.

13. Choi, J. -I., Yoon, M., Lim, S., Kim, G. -H., \& Park, H. (2015). Effect of gamma irradiation on physiological and proteomic changes of Arctic Zygnema sp. (Chlorophyta, Zygnematales). Phycologia, 54(4), 333-341. DOI: $10.2216 / 14-106.1$. 